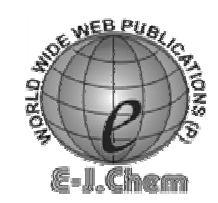

http://www.e-journals.net
ISSN: 0973-4945; CODEN ECJHAO

E-Journal of Chemistry

Vol. 5, No. 2, pp. 309-315, April 2008

\title{
Synthesis and Antimicrobial Studies of New Series of Pyrazoline Bearing Bis-Heterocycles via 1,3-Dipolar Cycloaddition Reactions
}

\author{
B. JAYASHANKARA and K.M. LOKANATHA RAI* \\ Department of Studies in Chemistry, University of Mysore, \\ Manasagangothri, Mysore-570006, India. \\ jai_shan321@yahoo.co.in
}

Received 12 September 2007; Revised 28 October 2007; Accepted 10 November 2007

\begin{abstract}
Biologically interesting bis-heterocycles bearing pyrazoline and imidazole moieties have been synthesized. ${ }^{1} \mathrm{H}$ NMR, ${ }^{13} \mathrm{C}$ NMR, IR and elemental analyses characterized the newly synthesized compounds. All the synthesized compounds were evaluated for their antimicrobial activity and were compared with the standard drugs. All the compounds demonstrated potent to weak antimicrobial activity.
\end{abstract}

Keywords: Synthesis; Bis-Heterocycle; Antimicrobial activity; Chloramine-T

\section{Introduction}

The development of simple, facile and efficient synthetic methods for the synthesis of five membered heterocycles from readily available reagents is one of the major challenges in organic synthesis. Among five membered heterocycles, pyrazoline and imidazole are represents a class of compounds of great importance in biological chemistry. For instance, pyrazoline derivatives posses the biological activities like, antidepressant ${ }^{1}$, anticonvulsant ${ }^{2}$ antimicrobial $^{3}$, analgesic ${ }^{4}$ and antitumour ${ }^{5}$ activity and also serves as human acyl-CoA: cholesterol acyltransferase inhibitors. ${ }^{6}$ In fact, celecoxib a pyrazole derivative is now widely used in the market as anti-inflammatory drug. ${ }^{7}$ Imidazole derivatives are gaining synthetic interest in recent years due to their broad spectrum of biological activities like antiinflammatory $^{8}$, analgesic ${ }^{9}$, antibacterial ${ }^{10}$, antifungal ${ }^{11}$, antituberculosis ${ }^{12}$, anticonvolusant ${ }^{13}$ and potential anticytokine agents ${ }^{14}$. 2-n-Butyl-4-chloro-5-farmyl-imidazole is a key intermediate for the synthesis of Losartan a nonpeptide angiotensin antagonist, which is an orally active antihypertensive drug ${ }^{15}$. Literature studies reveals that bis-heterocycles bearing pyrazoline ${ }^{16,17}$ were synthesized via 1,3-dipolar cycloaddition of aldehyde hydrazone to divinyl ketone / sulfone using chloramine-T (CAT) as dehydrating agent. 
1,3-Dipolar cycloaddition reactions are useful tools for constructing biologically potent five membered heterocycles ${ }^{18}$. Apart from the various dipolar reagents known, nitrile imines are used in numerous 1,3-dipolar cycloaddition reactions leading to pyrazoles, pyrazolines, pyrazolidines and other heterocyclic compounds ${ }^{19}$. Huisgen and co-workers ${ }^{20}$ first reported the authentic in situ generation of nitrile imines by the thermolysis of 2,5-diphenyl tetrazole in the presence of ethyl phenylpropionate and obtained 2,3,5-triphenyl carbethoxypyrazole. Nitrile imines can be generated by photolysis of sydnones ${ }^{21}$ and oxidation of aryl aldehyde hydrazones with lead tetraacetate ${ }^{22}$, chloramine-T ${ }^{23}$ etc. In our laboratory Rai et al extensively used chloramine-T for the generation of nitrile oxide and nitrile imine from aldoxime and aldehyde hydrazones respectively. With this background, it is considered worthwhile to prepare bis-heterocycles using 1,3-dipolar cycloaddition reaction of 2- $n$-butyl4-chloro-( $N$-substituted)-imidazole substituted nitrile imine with different olefins and screen them for antimicrobial activity. The present communication deals with the synthesis of bis-heterocycles via 1,3-dipolar cycloaddition reactions and their antimicrobial activity.

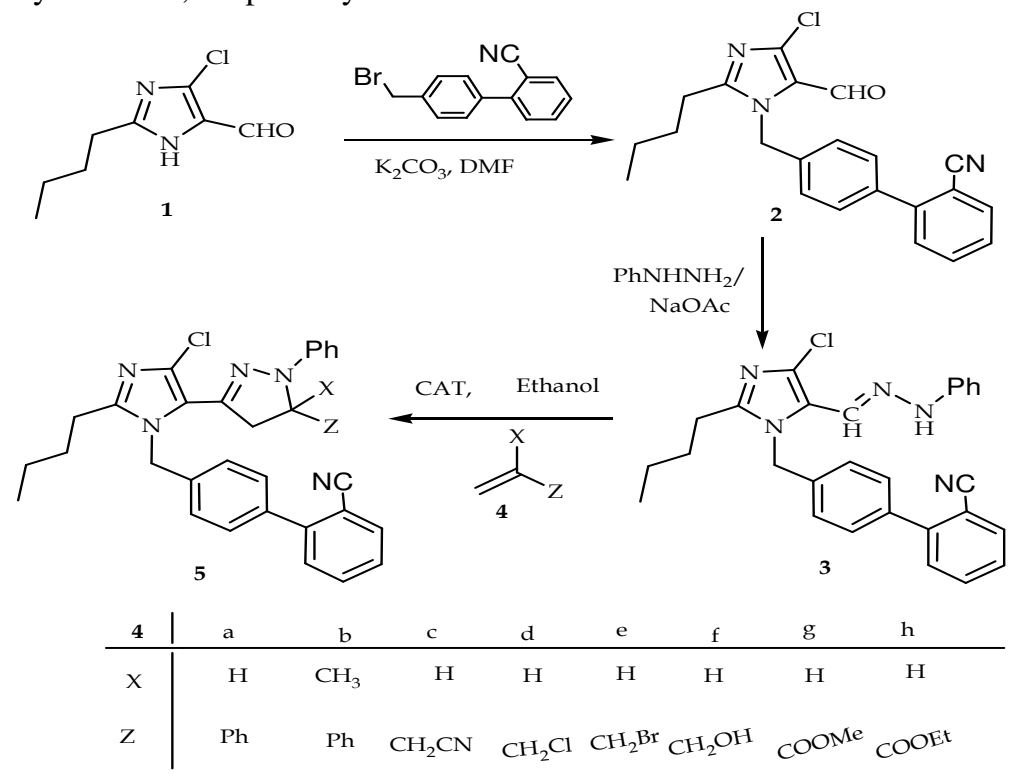

\section{Experimental}

Scheme 1

${ }^{1} \mathrm{H}$ NMR spectra were recorded on a Bruker AM $300 \mathrm{MHz}$ spectrometer using $\mathrm{CDCl}_{3}$ as solvent and tetramethylsilane as internal standard. ${ }^{13} \mathrm{C}$ NMR spectra were measured on Jeol $400(100 \mathrm{MHz})$ instrument. The chemical shifts are expressed in $\delta$ and following abbreviations were used: $\mathrm{s}=$ singlet, $\mathrm{d}=$ doublet, $\mathrm{t}=$ triplet and $\mathrm{m}=$ multiplet. Infrared (IR) spectra were measured on Shimadzu 8300 spectrometer. Elemental analyses were obtained on a Vaio-EL intrument. Thinlayer chromatography (TLC) was done with pre-coated silica gel $\mathrm{G}$ plates using benzene-ethylacetrate as eluent.

\section{Antimicrobial activity}

All the synthesized compounds were evaluated for antimicrobial activity by the disc diffusion method ${ }^{26}$ and microdilution method ${ }^{27}$. Five bacteria and five fungal species were 
used as the antimicrobial test strains namely: Bacillus substilis, Escherichia coli, Pseudomonas fluorescens, Xanthomonas campestris pvs, Xanthomonas oryzae, Aspergillus niger, Aspergillus flavus, Fusarium oxysporium, Trichoderma species, Fusarium and monaliforme. Streptomycin and tetracycline were used as standard drugs against bacteria and nystatin was used against fungi. In all the determinations tests were performed in triplicate and the results were taken as a mean of at least three determinations.

Preparation of 4'-(2-Butyl-4-chloro-5-formyl-imidazol-1-ylmethyl)-2-carbonitrile (2)

A mixture of 2-butyl-5-chloro-3H-imidazole-4-carbaldehyde $(1.0 \mathrm{~g}, 5.37 \mathrm{mmol})$ and anhyd. $\mathrm{K}_{2} \mathrm{CO}_{3}(0.90 \mathrm{~g}, 6.52 \mathrm{mmol})$ in dimethylformamide $(15 \mathrm{~mL})$ were stirred for $15 \mathrm{~min}$ at room temperature. 4'-Bromomethyl-biphenyl-2-carbonitrile (1.45 g, $5.33 \mathrm{mmol})$ was then added and the mixture was stirred at room temperature for $6 \mathrm{~h}$. After completion of the reaction (TLC toluene-ethylacetate; 7:3) the reaction mass was diluted with $25 \mathrm{~mL}$ water and the product was extracted with dichloromethane $(25 \mathrm{~mL})$. The extract was washed with water $(10 \mathrm{~mL})$ and then dried $\left(\mathrm{Na}_{2} \mathrm{SO}_{4}\right)$. The solvent was evaporated and the remaining pale yellow oil was crystallized from ethanol to give 2 as a white crystalline solid (1.81 g 89\%), mp $108-110^{\circ} \mathrm{C}$.

Synthesis of 4,5-dihydro-3-(substituted-imidazole)-1,5-diphenyl-1H-pyrazoline (5a)

A mixture of $\mathbf{3}(1.0 \mathrm{~g}, 2.13 \mathrm{mmol}), \mathbf{4 a}(0.23 \mathrm{~g}, 2.2 \mathrm{mmol})$ and chloramine-T trihydrate $(0.6 \mathrm{~g}, 2.13$ $\mathrm{mmol})$ in ethanol $(20 \mathrm{~mL})$ was warmed on a water bath for $2-3 \mathrm{~h}$. TLC monitored the progress of the reaction. After completion of the reaction the solvent was evaporated in vacuum. The residual mass was extracted into ether $(25 \mathrm{~mL})$, washed successively with water $(2 \times 20 \mathrm{~mL}), 5 \% \mathrm{NaOH}$ $(1 \times 10 \mathrm{~mL})$, brine solution $(2 \times 15 \mathrm{~mL})$ and dried over anhydrous sodium sulphate. Evaporation of the solvent afforded crude oily substance, which was purified by column chromatography using benzene-ethylacetate (8:1) as eluent to give the product as thick oil $(0.75 \mathrm{~g}, 62 \%)$.

\section{4,5-dihydro-3-(substituted-imidazole)-5-methyl-1,5-diphenyl-1H-pyrazoline (5b)}

Obtained from 3 (1.0 g, $2.13 \mathrm{mmol}), 4 \mathbf{b}(0.25 \mathrm{~g}, 2.12 \mathrm{mmol})$ and chloramine-T trihydrate $(0.6 \mathrm{~g}, 2.13 \mathrm{mmol})$ as thick oil $(0.88 \mathrm{~g}, 70 \%)$.

\section{4,5-dihydro-3-(substituted-imidazole)-1-phenyl-1H-pyrazoline-5-carbonitrile (5c)}

Obtained from 3 (1.0 g, $2.13 \mathrm{mmol}), 4 \mathbf{c}(0.12 \mathrm{~g}, 2.20 \mathrm{mmol})$ and chloramine-T trihydrate (0.6 g, $2.13 \mathrm{mmol})$ as thick oil $(0.7 \mathrm{~g}, 63 \%)$.

\section{5-(Chloromethyl)-4,5-dihydro-3-(substituted-imidazole)-1-phenyl-1H-pyrazoline (5d)}

Obtained from $3(1.0 \mathrm{~g}, 2.13 \mathrm{mmol}), \mathbf{4 d}(0.17 \mathrm{~g}, 2.23 \mathrm{mmol})$ and chloramine-T trihydrate $(0.6 \mathrm{~g}, 2.13 \mathrm{mmol})$ as thick oil $(0.73 \mathrm{~g}, 63 \%)$.

\section{5-(bromomethyl)-4,5-dihydro-3-(substituted-imidazole)-1-phenyl-1H-pyrazoline (5e)}

Obtained from 3 (1.0 g, $2.13 \mathrm{mmol}), 4 \mathrm{e}(0.26 \mathrm{~g}, 2.14 \mathrm{mmol})$ and chloramine-T trihydrate $(0.6 \mathrm{~g}, 2.13 \mathrm{mmol})$ as thick oil $(0.82 \mathrm{~g}, 66 \%)$.

\section{4,5-dihydro-3-(substituted-imidazole)-1-phenyl-1H-pyrazol-yl)methanol (5f)}

Obtained from $3(1.0 \mathrm{~g}, 2.13 \mathrm{mmol}), \mathbf{4 f}(0.125 \mathrm{~g}, 2.15 \mathrm{mmol})$ and chloramine-T trihydrate $(0.6 \mathrm{~g}, 2.13 \mathrm{mmol})$ as thick oil $(0.68 \mathrm{~g}, 61 \%)$.

\section{4,5-dihydro-3-(substituted-imidazole)-1-phenyl-1H-pyrazol-5-yl acetate $\mathbf{( 5 g})$}

Obtained from 3 (1.0 g, $2.13 \mathrm{mmol}), 4 \mathrm{~g}(0.185 \mathrm{~g}, 2.15 \mathrm{mmol})$ and chloramine-T trihydrate $(0.6 \mathrm{~g}, 2.13 \mathrm{mmol})$ as thick oil $(0.91 \mathrm{~g}, 77 \%)$. 
4,5-dihydro-3-(substituted-imidazole)-1-phenyl-1H-pyrazol-5-yl propionate (5h)

Obtained from 3 (1.0 g, $2.13 \mathrm{mmol}), 4 \mathrm{~h}(0.215 \mathrm{~g}, 2.15 \mathrm{mmol})$ and chloramine-T trihydrate $(0.6 \mathrm{~g}, 2.13 \mathrm{mmol})$ as thick oil $(0.92 \mathrm{~g}, 76 \%)$.

\section{Results and Discussion}

The general synthetic pathway discussed hereafter is depicted in the Scheme. Compound $\mathbf{1}$ was alkylated using 4'-Bromomethyl-biphenyl-2-carbonitrile and potassium carbonate in DMF. Then farmyl function of $\mathbf{2}$ was converted into the phenylhydrazone $\mathbf{3}$. When oxidative dehydrogenation of $\mathbf{3}$ by chloramine-T (CAT) afforded nitrile imine, which was in situ trapped by the different olefins $4(\mathbf{a}-\mathbf{h})$ under refluxing condition in ethanol. Thus produced compound was identified by NMR spectroscopy and elemental analyses as 4,5-dihydro-3(substituted imidazole)-5-substituted-1-phenyl-1H-pyrazoline 5 (a-h) in good quality and yield. The starting substrate 2-n-butyl-4-chloro-(N-substituted)-imidazole-5-carbaldehyde $\mathbf{1}$ was prepared according to literature procedure ${ }^{24}$. Imidazole aldehyde phenylhydrazone was prepared by known procedure ${ }^{25}$.

\section{Antimicrobial activity}

Antimicrobial activity of all the compounds was shown in Table 1 and 2. Among the series of synthesized compounds, $\mathbf{5 d}$ and $\mathbf{5 e}$ shown better inhibition. Remaining compounds shown moderate inhibition. The better inhibition shown by $\mathbf{5 d}$ and $\mathbf{5 e}$ may be due to the presence of chloro and bromo group in the compound.

Table 1. Minimal inhibitory concentration in $\mu \mathrm{g} \mathrm{mL} L^{-1}[\mathbf{X}]$ and Inhibitory zone in (diameter) $\mathrm{mm}[\mathbf{Y}]$ of the synthesized compounds against tested bacterial strains by micro dilution method and disk diffusion method respectively

\begin{tabular}{|c|c|c|c|c|c|c|c|c|c|c|}
\hline \multirow{2}{*}{ Compound } & \multicolumn{2}{|c|}{$\begin{array}{l}\text { Bacillus } \\
\text { substilis } \\
\end{array}$} & \multicolumn{2}{|c|}{$\begin{array}{c}\text { Escherichia } \\
\text { coli }\end{array}$} & \multicolumn{2}{|c|}{$\begin{array}{l}\text { Pseudomonas } \\
\text { fluorescens }\end{array}$} & \multicolumn{2}{|c|}{$\begin{array}{l}\text { Xanthomonas } \\
\text { campestris pvs }\end{array}$} & \multicolumn{2}{|c|}{$\begin{array}{c}\text { Xanthomonas } \\
\text { oryzae }\end{array}$} \\
\hline & $\mathrm{X}$ & $\begin{array}{c}\mathrm{Y} \\
\mathrm{mm}\end{array}$ & $\mathrm{X}$ & $\begin{array}{c}\mathrm{Y} \\
\mathrm{mm}\end{array}$ & $X$ & $\begin{array}{c}\mathrm{Y} \\
\mathrm{mm}\end{array}$ & $\mathrm{X}$ & $\begin{array}{c}\mathrm{Y} \\
\mathrm{mm}\end{array}$ & $X$ & $\begin{array}{c}\mathrm{Y} \\
\mathrm{mm}\end{array}$ \\
\hline $5 a$ & 21 & 07 & 26 & 13 & 23 & 16 & 25 & 12 & 23 & 12 \\
\hline $5 b$ & 22 & 10 & 21 & 11 & 25 & 14 & 22 & 11 & 24 & 11 \\
\hline $5 \mathrm{c}$ & 19 & 13 & 17 & 14 & 20 & 17 & 19 & 14 & 23 & 12 \\
\hline $5 d$ & 17 & 14 & 12 & 13 & 13 & 15 & 10 & 10 & 11 & 10 \\
\hline $5 e$ & 18 & 11 & 14 & 11 & 12 & 16 & 12 & 11 & 14 & 12 \\
\hline $5 f$ & 25 & 07 & 22 & 09 & 28 & 13 & 27 & 10 & 23 & 11 \\
\hline $5 \mathrm{~g}$ & 22 & 10 & 22 & 10 & 26 & 11 & 23 & 11 & 24 & 12 \\
\hline $5 \mathrm{~h}$ & 24 & 09 & 22 & 09 & 29 & 15 & 25 & 12 & 23 & 10 \\
\hline Streptomycin & 19 & 13 & 13 & 14 & 12 & 17 & - & - & - & - \\
\hline Tetracycline & - & & - & & - & & 09 & 12 & 13 & 12 \\
\hline
\end{tabular}

Streptomycin sulfate ( $25 \mu \mathrm{g}$ per disc); Tetracycline $(25 \mu \mathrm{g}$ per disc) were used as positive reference standard antibactierial discs, Synthesized compounds ( $25 \mu \mathrm{g}$ per disc). 
Table 2. Minimal inhibitory concentration in $\mu \mathrm{g} \mathrm{mL} L^{-1}[\mathrm{X}]$ and Inhibitory zone in (diameter) $\mathrm{mm}[\mathrm{Y}]$ of the synthesized compounds against tested fungal strains by micro dilution method and disk diffusion method respectively

\begin{tabular}{|c|c|c|c|c|c|c|c|c|c|c|}
\hline \multirow[b]{2}{*}{ Compound } & \multicolumn{2}{|c|}{$\begin{array}{c}\text { Aspergillus } \\
\text { niger }\end{array}$} & \multicolumn{2}{|c|}{$\begin{array}{c}\text { Aspergillus } \\
\text { flavus }\end{array}$} & \multicolumn{2}{|c|}{$\begin{array}{c}\text { Fusarium } \\
\text { oxysporium }\end{array}$} & \multicolumn{2}{|c|}{$\begin{array}{c}\text { Trichoderma } \\
\text { species }\end{array}$} & \multicolumn{2}{|c|}{$\begin{array}{l}\text { Fusarium } \\
\text { monalifome }\end{array}$} \\
\hline & $X$ & $\begin{array}{c}\mathrm{Y} \\
\mathrm{mm}\end{array}$ & $X$ & $\begin{array}{c}\mathrm{Y} \\
\mathrm{mm}\end{array}$ & $X$ & $\begin{array}{c}\mathrm{Y} \\
\mathrm{mm}\end{array}$ & X & $\begin{array}{c}\mathrm{Y} \\
\mathrm{mm}\end{array}$ & $X$ & $\begin{array}{c}\mathrm{Y} \\
\mathrm{mm}\end{array}$ \\
\hline $5 a$ & 18 & 08 & 18 & 09 & 14 & 12 & 15 & 14 & 14 & 11 \\
\hline $5 b$ & 18 & 07 & 18 & 07 & 18 & 11 & 17 & 12 & 15 & 09 \\
\hline $5 c$ & 16 & 08 & 16 & 10 & 13 & 13 & 14 & 15 & 11 & 11 \\
\hline $5 d$ & 14 & 09 & 13 & 12 & 10 & 16 & 11 & 17 & 10 & 14 \\
\hline $5 e$ & 15 & 09 & 15 & 11 & 11 & 14 & 13 & 16 & 10 & 12 \\
\hline $5 f$ & 19 & 07 & 19 & 07 & 17 & 11 & 17 & 12 & 15 & 09 \\
\hline $5 \mathrm{~g}$ & 21 & 07 & 26 & 08 & 23 & 15 & 25 & 12 & 23 & 12 \\
\hline $5 \mathrm{~h}$ & 22 & 08 & 21 & 10 & 25 & 14 & 22 & 11 & 24 & 11 \\
\hline Nystatin & 15 & 08 & 15 & 10 & 11 & 14 & 12 & 16 & 09 & 12 \\
\hline
\end{tabular}

Nystatin $(25 \mu \mathrm{g}$ per disc) was used as positive reference standard antifungal discs, synthesized compounds ( $25 \mu \mathrm{g}$ per disc).

Spectral analysis of compounds

Compound 2: ${ }^{1} \mathrm{H}$ NMR CDCl${ }_{3}: \delta 0.92\left(\mathrm{t}, 3 \mathrm{H}, \mathrm{CH}_{3}\right), 1.34\left(\mathrm{~m}, 2 \mathrm{H}, \mathrm{CH}_{2}\right), 1,66\left(\mathrm{~m}, 2 \mathrm{H}, \mathrm{CH}_{2}\right), 2.57(\mathrm{t}$, $\left.2 \mathrm{H}, \mathrm{CH}_{2}\right), 4.96\left(\mathrm{~s}, 2 \mathrm{H}, \mathrm{CH}_{2}\right), 7.12-7.36(\mathrm{~m}, 4 \mathrm{H}, \mathrm{ArH}), 7.5-7.65(\mathrm{~m}, 4 \mathrm{H}, \mathrm{ArH}), 9.62(\mathrm{~s}, 1 \mathrm{H}, \mathrm{CH}),{ }^{13} \mathrm{C}$ NMR CDCl ${ }_{3}: \delta 14.2(\mathrm{C}), 23.4(\mathrm{C}), 23.9(\mathrm{C}), 34.8$ (C), 42.4 (C), 114.6 (C), $118.5(\mathrm{C}), 127.4(2 \mathrm{C})$, 128.2 (2C), 129.6 (2C), 132.1 (C), 133.2 (C), 133.4 (C), 136.1 (C), 136.9 (C), 140.9 (C), 142.9 (C), 161.7 (C), 189.1 (C). IR (KBr pellets $\left.\mathrm{cm}^{-1}\right)$ v 3070, .2996, 2241, 1762, 1667, 1576, 1299. Anal. Calcd. For $\mathrm{C}_{22} \mathrm{H}_{20} \mathrm{ClN}_{3} \mathrm{O}$ : C, 69.93, H, 5.33, N, 11.12\%. Found: C, 69.99, H, 5.38, N11.09\%.

Compound 5a: ${ }^{1} \mathrm{H} \mathrm{NMR} \mathrm{CDCl}_{3}: \delta 0.94\left(\mathrm{t}, 3 \mathrm{H}, \mathrm{CH}_{3}\right), 1.34\left(\mathrm{~m}, 2 \mathrm{H}, \mathrm{CH}_{2}\right), 1,66\left(\mathrm{~m}, 2 \mathrm{H}, \mathrm{CH}_{2}\right), 2.57$ (t, 2H, $\left.\mathrm{CH}_{2}\right), 3.34$ (dd, J=6.2, 1H, 4-H), 3.42 (dd, 1H, J=6.2, 4-H), 5.17 (dd, 1H, J=2.0, 5-H), 5.02 (s, 2H, $\left.\mathrm{CH}_{2}\right), 6.62-7.10(\mathrm{~m}, 5 \mathrm{H}, \mathrm{ArH}), 7.18-7.37$ (m, 4H, ArH), 7.42-7.65 (m, 4H, ArH), ${ }^{13} \mathrm{C}$ NMR $\mathrm{CDCl}_{3}: \delta 14.1(\mathrm{C}), 23.1(\mathrm{C}), 26.7(\mathrm{C}), 33.2(\mathrm{C}), 39.5(\mathrm{C}), 53.2(\mathrm{C}), 104.7(\mathrm{C}), 113.5(2 \mathrm{C}), 115.9(\mathrm{C})$, 117.7 (C), $122.2(\mathrm{C}), 126.3$ (C), 126.8 (C), $127.2(2 \mathrm{C}), 127.8$ (2C), 128.4 (C), 128.7 (4C), 129.7 (4C), 132.9 (C), 133.8 (2C), 135.4 (C), 142.7 (C), 143.4 (C), 143.8 (C), 148.6 (C), 156.1 (C). Anal.Calcd. For $\mathrm{C}_{36} \mathrm{H}_{32} \mathrm{ClN}_{5}$; C, 75.84; H, 5.66; N, 12.28; Found: C, 75.85, H, 5.67, N, 12.28

Compound 5b: ${ }^{1} \mathrm{H} \mathrm{NMR} \mathrm{CDCl}_{3}: \delta 0.96\left(\mathrm{t}, 3 \mathrm{H}, \mathrm{CH}_{3}\right), 1.36\left(\mathrm{~m}, 2 \mathrm{H}, \mathrm{CH}_{2}\right), 1.62\left(\mathrm{~s}, 3 \mathrm{H}, \mathrm{CH}_{3}\right), 1.65$ (m, 2H, $\left.\mathrm{CH}_{2}\right), 2.58\left(\mathrm{t}, 2 \mathrm{H}, \mathrm{CH}_{2}\right), 3.32\left(\mathrm{~s}, 2 \mathrm{H}, 4-\mathrm{CH}_{2}\right), 5.10\left(\mathrm{~s}, 2 \mathrm{H}, \mathrm{CH}_{2}\right), 6.64-7.06(\mathrm{~m}, 6 \mathrm{H}, \mathrm{ArH})$, 7.13-7.19 (m, 6H, ArH), 7.38-7.68 (m, 6H, ArH). ${ }^{13} \mathrm{C} \mathrm{NMR} \mathrm{CDCl}_{3}: \delta 14.2(\mathrm{C}), 23.2(\mathrm{C}), 26.8$ (C), 30.1 (C), 33.4 (C), 41.4 (C), 47.3 (C), 56.2 (C), 104.6 (C), 113.5 (2C), 115.9 (C), 117.8 (C), 122.2 (C), 126.2 (C), 126.2 (C), 126.6 (2C), 127.7 (2C), 128.4 (3C), 128.7 (C), 129.9 (4C), 132.9 (C), 133.4 (C), 133.8 (C), 135.4 (C), 142.7 (C), 143.8 (C), 144.4 (C), 148.3 (C), 156.3 (C). Anal.Calcd. For $\mathrm{C}_{37} \mathrm{H}_{34} \mathrm{ClN}_{5}$; C, 76.08; H, 5.87; N, 11.99; Found: C, 76.10, H, 5.86, N, $11.98 \%$.

Compound 5c: ${ }^{1} \mathrm{H}$ NMR $\mathrm{CDCl}_{3}: \delta 0.94\left(\mathrm{t}, 3 \mathrm{H}, \mathrm{CH}_{3}\right), 1.33\left(\mathrm{~m}, 2 \mathrm{H}, \mathrm{CH}_{2}\right), 1,64\left(\mathrm{~m}, 2 \mathrm{H}, \mathrm{CH}_{2}\right)$, 2.55 (t, $2 \mathrm{H}, \mathrm{CH}_{2}$ ), 3.37 (dd, 1H, J=6.0, 4-H), 3.40 (dd, 1H, J=6.0, 4-H), 5.19 (dd, 1H, J=3.6, 5$\mathrm{H}), 5.00\left(\mathrm{~s}, 2 \mathrm{H}, \mathrm{CH}_{2}\right), 6.60-7.08(\mathrm{~m}, 5 \mathrm{H}, \mathrm{ArH}), 7.16-7.32(\mathrm{~m}, 4 \mathrm{H}, \mathrm{ArH}), 7.40-7.65(\mathrm{~m}, 4 \mathrm{H}, \mathrm{ArH})$, ${ }^{13} \mathrm{C} \mathrm{NMR} \mathrm{CDCl}_{3}: \delta 14.2(\mathrm{C}), 23.0(\mathrm{C}), 25.7(\mathrm{C}), 32.6(\mathrm{C}), 33.5(\mathrm{C}), 40.8(\mathrm{C}), 41.1(\mathrm{C}), 104.7(\mathrm{C})$, 113.7 (2C), 115.9 (C), $116.6(\mathrm{C}), 117.8$ (C), 122.3 (C), 126.3 (C), 127.9 (2C), 128.5 (C), 128.8(C), 
129.7 (4C), 132.8 (C) 133.6 (2C), 135.4 (C), 142.7 (C), 144.0 (C), 148.5 (C), 156.7 (C). Anal.Calcd. For $\mathrm{C}_{31} \mathrm{H}_{27} \mathrm{ClN}_{6} ; \mathrm{C}, 71.73 ; \mathrm{H}, 5.24 ; \mathrm{N}, 16.19$. Found: C, 71.73; H, 5.23; N, $16.19 \%$.

Compound 5d: ${ }^{1} \mathrm{H}$ NMR CDCl 3 : $\delta 0.98\left(\mathrm{t}, 3 \mathrm{H}, \mathrm{CH}_{3}\right), 1.35\left(\mathrm{~m}, 2 \mathrm{H}, \mathrm{CH}_{2}\right), 1,66\left(\mathrm{~m}, 2 \mathrm{H}, \mathrm{CH}_{2}\right)$, 2.57 (t, $2 \mathrm{H}, \mathrm{CH}_{2}$ ), 3.32 (dd, 1H, J=6.4, 4-H), 3.37 (dd, 1H, J=6.4, 4-H), 3.46 (dd, 1H, J=4.0, $\left.\mathrm{CH}_{2} \mathrm{Cl}\right), 3.70\left(\mathrm{dd}, 1 \mathrm{H}, J=4.0, \mathrm{CH}_{2} \mathrm{Cl}\right), 4.98\left(\mathrm{~s}, 2 \mathrm{H}, \mathrm{CH}_{2}\right), 5.10(\mathrm{~m}, 1 \mathrm{H}, 5-\mathrm{H}), 6.58-7.08(\mathrm{~m}$, $5 \mathrm{H}, \mathrm{ArH}), 7.16-7.38(\mathrm{~m}, 4 \mathrm{H}, \mathrm{ArH}), 7.42-7.65(\mathrm{~m}, 4 \mathrm{H}, \mathrm{ArH}),{ }^{13} \mathrm{C} \mathrm{NMR} \mathrm{CDCl}_{3}: \delta 14.2(\mathrm{C})$, 22.8 (C), 26.0 (C), 33.2 (C), 34.6 (C), 40.7 (C), 52.4 (C), 53.4 (C), 104.7 (C), 113.6 (2C), 116.0 (C), 117.5 (C), 122.2 (C), 126.3 (C), 127.8 (2C), 128.4 (C), 128.8 (C), 129.7 (4C), 132.4 (C), 133.5 (2C), 135.4 (C), 142.5 (C), 144.0 (C), 148.4 (C), 156.4 (C). Anal.Calcd. For $\mathrm{C}_{31} \mathrm{H}_{29} \mathrm{Cl}_{2} \mathrm{~N}_{5} ; \mathrm{C}, 68.63 ; \mathrm{H}, 5.39 ; \mathrm{N}, 12.91$. Found: C, 68.64; H, 5.38; N, $12.93 \%$.

Compound 5e: ${ }^{1} \mathrm{H} \mathrm{NMR} \mathrm{CDCl}_{3}: \delta 0.95\left(\mathrm{t}, 3 \mathrm{H}, \mathrm{CH}_{3}\right), 1.32\left(\mathrm{~m}, 2 \mathrm{H}, \mathrm{CH}_{2}\right), 1,63\left(\mathrm{~m}, 2 \mathrm{H}, \mathrm{CH}_{2}\right)$, $2.54\left(\mathrm{t}, 2 \mathrm{H}, \mathrm{CH}_{2}\right), 3.30(\mathrm{dd}, 1 \mathrm{H}, J=6.6,4-\mathrm{H}), 3.35(\mathrm{dd}, 1 \mathrm{H}, J=6.6,4-\mathrm{H}), 3.42(\mathrm{dd}, 1 \mathrm{H}, J=3.2$, $\mathrm{CH}_{2} \mathrm{Br}$ ), 3.68 (dd, $1 \mathrm{H}, J=3.2, \mathrm{CH}_{2} \mathrm{Br}$ ), $5.16(\mathrm{~m}, 1 \mathrm{H}, 5-\mathrm{H}), 4.98$ (s, $\left.2 \mathrm{H}, \mathrm{CH}_{2}\right), 6.50-7.08$ (m, 5H, $\mathrm{ArH}), 7.12-7.36(\mathrm{~m}, 4 \mathrm{H}, \mathrm{ArH}), 7.42-7.65(\mathrm{~m}, 4 \mathrm{H}, \mathrm{ArH}),{ }^{13} \mathrm{C} \mathrm{NMR} \mathrm{CDCl}_{3}: \delta 14.2(\mathrm{C}), 22.6(\mathrm{C})$, 26.0 (C), 33.2 (C), 35.9 (C), 36.6 (C), 39.2 (C), 40.5 (C), 54.4 (C), 104.7 (C), 113.6 (2C), 116.0 (C), $117.4(\mathrm{C}), 122.0(\mathrm{C}), 126.2(\mathrm{C}), 127.8$ (2C), 128.4 (C), 128.7 (C), $129.7(4 \mathrm{C}), 132.5$ (C), 133.5 (C), 135.4 (C), 142.6 (C), 143.8 (C), 148.2 (C), 155.4 (C). Anal.Calcd. For $\mathrm{C}_{31} \mathrm{H}_{29} \mathrm{BrClN}_{5}$; C, 63.43; H, 4.98; N, 11.93. Found: C, 63.45, H, 4.98, N, $11.91 \%$.

Compound 5f: ${ }^{1} \mathrm{H}$ NMR CDCl $3: \delta 0.96\left(t, 3 \mathrm{H}, \mathrm{CH}_{3}\right), 1.33\left(\mathrm{~m}, 2 \mathrm{H}, \mathrm{CH}_{2}\right), 1,63\left(\mathrm{~m}, 2 \mathrm{H}, \mathrm{CH}_{2}\right), 2.56$ (t, $\left.2 \mathrm{H}, \mathrm{CH}_{2}\right), 3.24$ (dd, $\left.1 \mathrm{H}, J=6.2,4-\mathrm{H}\right), 3.30$ (dd, 1H, J=6.2, 4-H), 3.61-3.86 (m, 2H, $\left.\mathrm{CH}_{2}\right), 5.02$ (s, $\left.2 \mathrm{H}, \mathrm{CH}_{2}\right), 5.17(\mathrm{~m}, 1 \mathrm{H}, 5-\mathrm{H}), 6.52-7.10(\mathrm{~m}, 5 \mathrm{H}, \mathrm{ArH}), 7.16-7.37$ (m, 4H, ArH), 7.42-7.65 (m, 4H, $\mathrm{ArH}) .{ }^{13} \mathrm{C}$ NMR CDCl 3 : $\delta 14.3(\mathrm{C}), 23.1(\mathrm{C}), 26.0(\mathrm{C}), 33.2(\mathrm{C}), 33.7(\mathrm{C}), 40.9(\mathrm{C}), 53.1(\mathrm{C}), 66.4$ (C), 104.5 (C), 113.5 (2C), 115.8 (C), 117.2 (C), 122.2 (C), 126.7 (C), 127.7 (2C), 128.5 (C), 128.9 (C), 129.7 (4C), 132.8 (C), 133.3 (C), 133.8 (C), 135.4 (C), 142.6 (C), 144.0 (C), 148.4 (C), 156.5 (C). Anal.Calcd. For $\mathrm{C}_{31} \mathrm{H}_{30} \mathrm{ClN}_{5} \mathrm{O}$; C, 71.05; H, 5.77; N, 13.36; Found: C, 69.99, H, 6.13, N, 13.03 .

Compound 5g: ${ }^{1} \mathrm{H}$ NMR $\mathrm{CDCl}_{3}: \delta 0.94\left(\mathrm{t}, 3 \mathrm{H}, \mathrm{CH}_{3}\right), 1.33\left(\mathrm{~m}, 2 \mathrm{H}, \mathrm{CH}_{2}\right), 1,66\left(\mathrm{~m}, 2 \mathrm{H}, \mathrm{CH}_{2}\right)$, $2.02\left(\mathrm{~s}, 3 \mathrm{H}, \mathrm{CH}_{3}\right), 2.55\left(\mathrm{t}, 2 \mathrm{H}, \mathrm{CH}_{2}\right), 3.37(\mathrm{dd}, 1 \mathrm{H}, J=6.0,4-\mathrm{H}), 3.42(\mathrm{dd}, 1 \mathrm{H}, J=6.0,4-\mathrm{H})$, $5.42(\mathrm{dd}, 1 \mathrm{H}, J=4.0,5-\mathrm{H}), 5.04\left(\mathrm{~s}, 2 \mathrm{H}, \mathrm{CH}_{2}\right), 6.57-7.06(\mathrm{~m}, 5 \mathrm{H}, \mathrm{ArH}), 7.15(\mathrm{~d}, 2 \mathrm{H}), 7.37$ (d, 2H, ArH), 7.42-7.67 (m, 4H, ArH), ${ }^{13} \mathrm{C} \mathrm{NMR} \mathrm{CDCl}{ }_{3}: \delta 14.3(\mathrm{C}), 20.8(\mathrm{C}), 23.3(\mathrm{C}), 26.7$ (C), 33.5 (C), 37.2 (C), 40.6 (C), 78.4 (C), 104.7 (C), 113.6 (2C), 115.9 (C), 117.3 (C), 122.1 (C), 126.6 (C), 127.8 (2C), 128.4 (C), 128.8 (C), 129.6 (4C), 132.7 (C), 133.5 (C), 133.8 (C), 135.4 (C), 142.7 (C), 144.1 (C), 148.3 (C), 155.9 (C), 170.5 (C). Anal.Calcd. For $\mathrm{C}_{32} \mathrm{H}_{30} \mathrm{ClN}_{5} \mathrm{O}_{2} \mathrm{C}, 69.62 ; \mathrm{H}, 5.48$; N, 12.69; Found: C, 69.62, H, 5.46, N, $12.70 \%$.

Compound 5h: ${ }^{1} \mathrm{H}$ NMR $\mathrm{CDCl}_{3}: \delta 0.96\left(\mathrm{t}, 3 \mathrm{H}, \mathrm{CH}_{3}\right), 1.14\left(\mathrm{t}, 3 \mathrm{H}, \mathrm{CH}_{3}\right), 1.34\left(\mathrm{~m}, 2 \mathrm{H}, \mathrm{CH}_{2}\right), 1,66$ (m, $2 \mathrm{H}, \mathrm{CH}_{2}$ ), 2.32 (q, 2H, $\mathrm{CH}_{2}$ ), 2.57 (t, 2H, $\mathrm{CH}_{2}$ ), 3.34 (dd, 1H, J=6.4, 4-H), 3.42 (dd, 1H, $J=6.4,4-\mathrm{H}), 5.38$ (dd, $1 \mathrm{H}, J=4.0,5-\mathrm{H}), 5.02\left(\mathrm{~s}, 2 \mathrm{H}, \mathrm{CH}_{2}\right), 6.52-7.05(\mathrm{~m}, 5 \mathrm{H}, \mathrm{ArH}), 7.14-7.37$ (m, 4H, ArH), 7.40-7.66 (m, 4H, ArH), ${ }^{13} \mathrm{C} \mathrm{NMR} \mathrm{CDCl}$ : $\delta 10.1$ (C), 14.1 (C), $22.9(\mathrm{C}), 25.7$ (C), 27.6 (C), 33.7 (C), 37.5 (C), 40.7 (C), 50.1 (C), 78.9 (C), 104.7 (C), 113.6 (2C), 115.8 (C), 117.7 (C), 122.3 (C), 126.7 (C), 127.8 (2C), 128.4 (C), 128.7 (C), 129.7 (4C), 132.8 (C), 133.5 (C), 135.4 (C), 142.7 (C), 144.0 (C), 148.4 (C), 156.8 (C), 173.2 (C). Anal.Calcd. For $\mathrm{C}_{33} \mathrm{H}_{32} \mathrm{ClN}_{5} \mathrm{O}_{2}$; C, 70.02; H, 5.70; N, 12.37; Found: C, 70.04, H, 5.70, N, $12.38 \%$.

\section{Conclusion}

In conclusion 4, 5- dihydro -3- (substituted - imidazole) - 5 - substituted-1-phenyl-1H-pyrazoline derivatives were synthesized and their antimicrobial activity have been evaluated. Compounds $\mathbf{5 d}$ and 5e shows significant inhibition, remaining compounds demonstrated potent to moderate antimicrobial activity. Further research in this area is under progress in our laboratory. 


\section{Acknowledgement}

One of the authors (B. J. Shankar) is grateful to Dr. S.L. Gaonkar providing me the imidazole aldehyde and university of Mysore for financial support.

\section{References}

1. Rajendra Prasad Y, Laxmana Rao L, Prasoona K, Murali and Ravi Kumar P. Bioorg. Med. Chem .Lett. 2005, 15, 5030.

2. Zuhal Ozdemir, Burak Kandilie H and Bulent Gumuse, Euro.J.Med.Chem. 2007, 42, 373.

3. Ahmet Ozdemir, Gulhan Turan-zitouni and Zafer Asim kaplaneikle, Euro. J. Med. Chem. 2007, 42, 403.

4. Aysel Gursoy S, Demirayak, Gultaze capen. et.al. Euro.J.Med.Chem. 2000, 35, 359.

5. Brzozwski Z, Czewski F S and Gdaniec. Euro.J.Med.Chem. 2000, 35, 1053.

6. Tae-Sook Jeong, Kyung Soon Kim, So-Jin An, Kyung-Hyun Cho, Sangku Lee and Woo Song Lee. Bioorg. Med. Chem .Lett. 2004, 14, 2715.

7. Dannahardt G, Kiefer W, Kramer G, Maehrlein S, Nowe U and Fiebich B, Eur. J. Med. Chem, 2000, 35, 499.

8. Slee D H, Romano S J, yu J, Nguyen T N, John J K, Raheja N K. Axe F U, Jones T K and Ripka W C, J. Med. Chem., 2001, 44, 2094.

9. Umit U, Nalan G Karaburun and Ihan L, II Farmaco, 2001, 56, 285.

10. Khabnadideh S, Rezaei Z, Khalafi-Nezhad A, Bahrinajafi R, Mahamadi R and Farrokhroz A A, Bioorg. Med. Chem. Lett. 2003, 13, 2863.

11. Gunnay N S, Ulusoy G N, Ergenc N, Otuk G and Kaya D, II Farmaco, 1999, 54, 826.

12. Gupta P, Hameed S and Jain R, Euro. J. Med. Chem., 2004, 39, 805.

13. Soyer Z, Sultan F, Erol K K and Pabuccuolu V, II Farmaco, 2004, 59, 595.

14. Laufer S A, Striegel H G and Wagner G K, J. Med. Chem., 1993, 45, 4695.

15. Carini D J, Duncia J V, Aldrich P E, Chiu A T, Johnson A L, Pierce M E, Price W A, Santella III J B, Wells G J, Wexler P C, Yoo S W and Timmermans P B M W M, J. Med. Chem., 1991, 34, 2525.

16. Padmavathi V, Jagan Mohan Reddy B, Chandra Obula Reddy B and Padmaja A. Tetrahedron, 2005, 61, 2407.

17. Padmavathi V, Venugopal Reddy K, Padmaja A, and Bhaskar Reddy D Phosphorus, Sulfur and Silicon, 2003, 178, 171.

18. Caramella $\mathrm{P}$ and Gruinanger $\mathrm{P}$ in 1,3-Dipolar Cycloaddition Chemistry, vol 1, Edited by Padwa A (Wiley Interscience, New york) 1984, 337.

19. Huisgen R, Seidel M, Sauer J, Mc Farland J M and Wallibillich G, J Org Chem. 1959, 24, 892.

20. Huisgen R, Seidel M, Wallibillich G and Knupfer H, Tetrahedron, 1962, 17, 3.

21. Marky M, Meier H, Wunderli A, Hemigarther H, Schmidt H and Hansen H J, Helv Chem Acta, 1978, 61, 1477.

22. Gladstone W A, Aylward J B and Norman R O C, J Chem Soc, (C), 1969, 2587.

23. Lokanath Rai K M and Hassner A, SynthCommun. 1989, 19, 2799.

24. Griffiths G J, Hauck M B, Imwinkelried R, Kohr J, Roten C A, Stucky G C, J. Org. Chem., 1999, 64, 8084.

25 Vogel. A. I., A Textbook of practical Organic Chemistry, $5^{\text {th }}$ Ed; Longman's Green and Co. Ltd: London, 1989; 1258.

26 Lemriss S, Marquet B, Ginestet H, Lefeuvre L, Fassouane A and Boiron P, J. Mycol. Med, 2003, 13, 189.

27. Zgoda J R and Porter J R, Pharmaceutical Biology, 2001, 39, 221. 


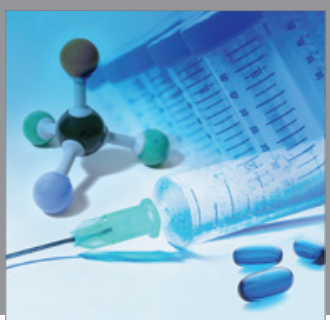

International Journal of

Medicinal Chemistry

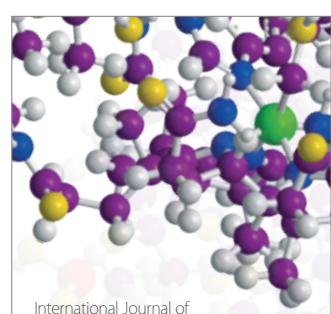

Carbohydrate Chemistry

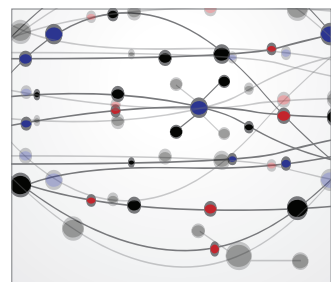

The Scientific World Journal
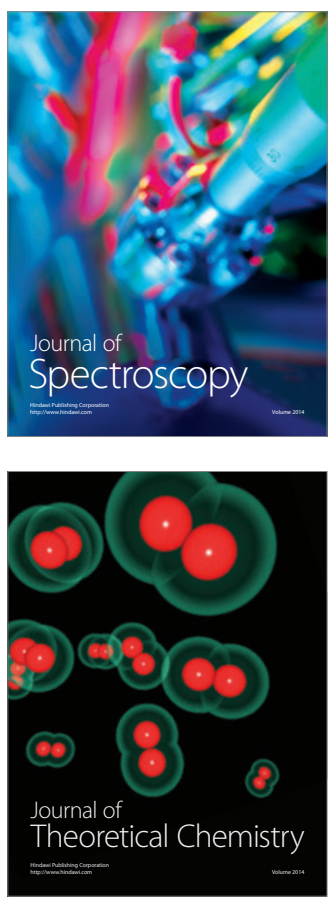
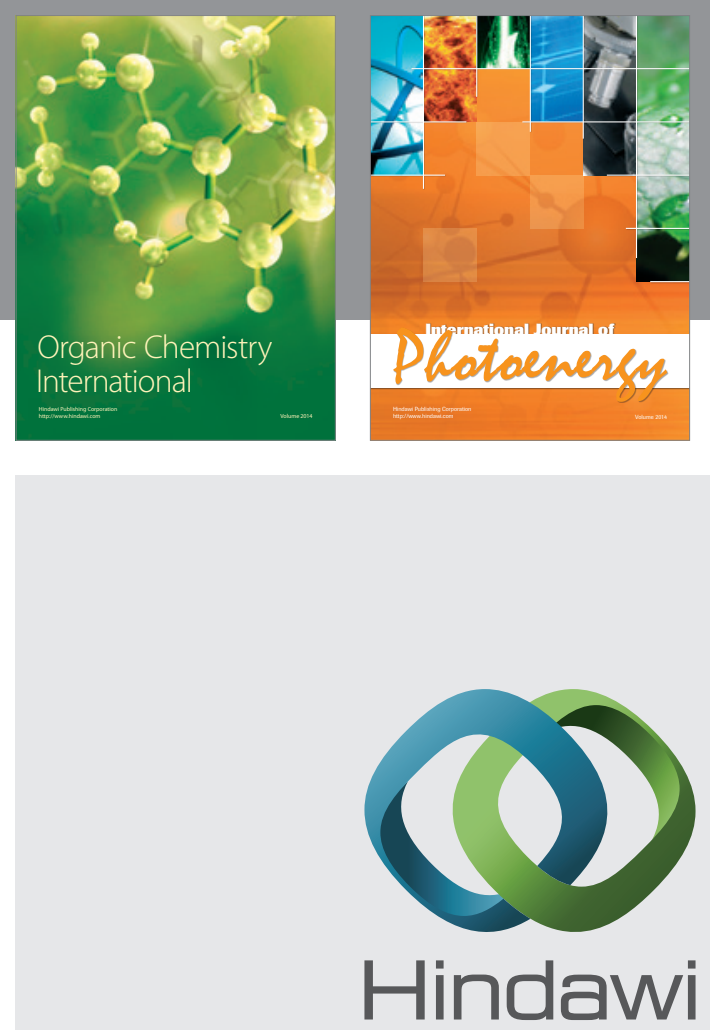

Submit your manuscripts at

http://www.hindawi.com
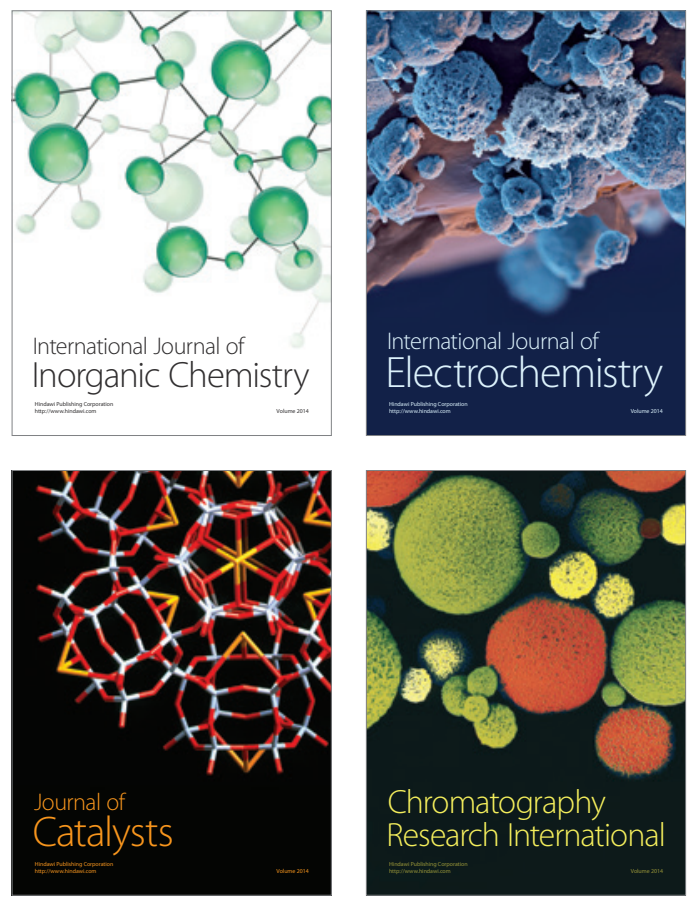
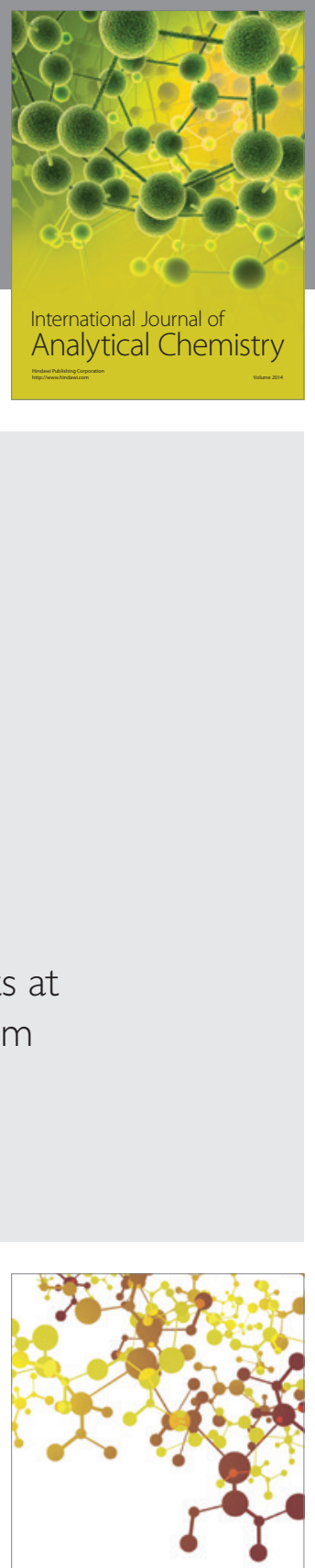

Journal of

Applied Chemistry
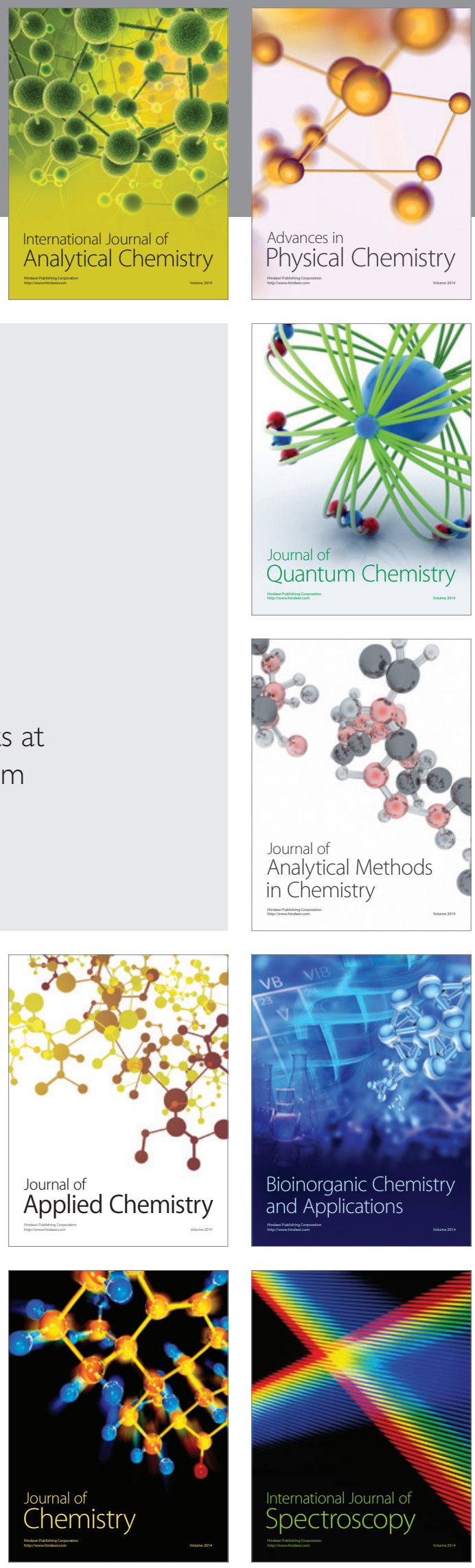